\title{
A cognitive load assessment study of three-dimensional interactive virtual reality interfaces
}

\author{
Akriti Kaur \\ Department of Design, \\ Indian Institute of Technology, \\ Guwahati 781039, India \\ Email: akriti978@gmail.com \\ *Corresponding author

\section{Mudit Agrawal} \\ Department of CSE, \\ Indian Institute of Technology, \\ Guwahati 781039, India \\ Email: muditag313@gmail.com
}

\section{Pradeep G. Yammiyavar}

Department of Design, Indian Institute of Technology, Guwahati 781039, India

Email: pradeep@iitg.ernet.in

\begin{abstract}
Advances in virtual reality (VR) open possibilities for novel three-dimensional (3D) graphical user interfaces (GUIs) to emerge for complex tasks and information visualisation. However, three dimensional GUIs do not have many design heuristics. Since 3D GUIs deal with a third dimension, understanding cognitive workload of such systems need to consider a set of different variables to make the interface more usable. Factors like the field of view, physical demand, and mental demand that increase the workload in interacting with three dimensional interfaces need to be considered. This paper reports an experiment by collating quantitative and qualitative responses from $N=30$ users to explore usability problems that are likely to be encountered when a two-dimensional (2D) interface element such as number keypad is replaced with a 3D element interface in VR. Would an interface with 3D elements perform better than the existing 2D GUIs is a moot research question? The results indicate user likeliness towards the three dimensional interface. The paper discusses issues of interaction in $2 \mathrm{D}$ and $3 \mathrm{D}$ virtual spaces with their possible implications for upcoming 3D VR environments.
\end{abstract}

Keywords: virtual reality; usability assessment; three-dimensional graphical user interfaces; GUIs; user-centred design.

Reference to this paper should be made as follows: Kaur, A., Agrawal, M. and Yammiyavar, P.G. (2020) 'A cognitive load assessment study of three-dimensional interactive virtual reality interfaces', Int. J. Forensic Engineering and Management, Vol. 1, No. 1, pp.103-115. 
Biographical notes: Akriti Kaur did her Bachelors in Design from IIT Guwahati, India. She was an active researcher in the Usability Lab, IITG performing various experimental studies and user research. She was able to secure ACM SIGGRAPH student travel grant to present her research. She is currently pursuing her Masters at the University of Toronto, Canada.

Mudit Agrawal is a Software Engineer. He completed his BTech from Indian Institute of Technology, Guwahati in Computer Science and Engineering. He currently works at Nutanix Inc. as a member of technical staff. He is an enthusiastic game developer and has developed some games on small scale like Scrabble in Hindi Language, Laser Tag Prototype using IoT, a volleyball game using kinect. He was also the secretary of Coding Club, IITG for a year. He also developed a prototype dial pad in VR which was helpful in assessment study of 3D Interactive Virtual Reality Interfaces.

Pradeep G. Yammiyavar is a Senior Professor of Design Research at the Indian Institute of Technology Guwahati, India with interest in human computer interaction design and product usability engineering. He is an Advisor to educational institutions in creative design disciplines including architecture and urban built environment. He is author of over 150 research publications and honoured with numerous awards both nationally and internationally - the most recent being a national award for Teaching Innovation for his contributions to design education and development of innovative interdisciplinary design curriculum. Besides being a designer and engineer, he is also a trained Psychologist in mental wellbeing. He is a Fellow of the Design Research Society, UK.

This paper is a revised and expanded version of a paper entitled 'A cognitive load assessment study of three-dimensional interactive virtual reality interfaces' presented at HWWE 2017, Aligarh, India, 8-10 December 2017.

\section{Introduction}

Virtual environments (VEs) have huge implications in home, school, workplace, medicine and several other areas. Enough research has been done in the field of development of technology in virtual reality (VR) and different input methods used for interaction with the three-dimensional (3D) space, but comparatively little has been studied on the usability and ergonomics aspects of VR (Nichols, 1999). In order to reduce cognitive load with 3D graphical user interface (GUI) in the VE, should we deploy the methods currently used in human computer interaction for two-dimensional (2D) interfaces, is a moot research question? Research has shown several problems associated with this approach. An important issue here is that HCI is a field that has evolved from command line to GUIs on a desktop screen, and then to interfaces involving different multimedia; is it justified to apply the same rules to a VE where the user navigates within and outside the VE? Will such guidelines be helpful to solve problems unique to VR, like temporal lags, field of view (FOV) of head mounted displays (HMDs) and complexity of the 3D interfaces? (Wilson, 1999). This paper discusses ergonomic issues related to a spatial mobile keypad interface in VR. The factors discussed in this study include:

1 FOV of the HMD 
2 Orientation issues of the user

3 utilisation of the third dimension - depth.

Based on the task performed in VR, a decision needs to be made about the FOV. Narrow FOVs may affect the task performances involving grasping objects and identifying moving objects in space. Designing wider FOVs may improve task performances and increase immersion in the VR environment, but they compromise with image resolution and need a larger HMD for their functioning. In a VE, the user can interact from within an environment, using natural semantics rather than menus and command lines. This type of interaction enhances user involvement and satisfaction, but also brings a lot of usability problems like making users understand their position in space, navigation in the $\mathrm{VE}$, wayfinding and orientation. In a VE, visual cues play a major role in user involvement. What approach should be followed in order to design visuals for 3D GUI? Research shows two possible approaches: to recreate the real environment or to create a $\mathrm{VE}$ that is closely relatable to reality, maybe with the use of metaphors and inspirations from the real world but not necessarily replicating it (Wilson, 1997). Thus, VE require redefining of 'traditional HCI' guidelines since the user interacts more intuitively from 'within' the space created via VE. This paper discusses an experiment to evaluate spatial mobile keypads inspired from 2D and 3D elements in VR.

\section{Background study}

VEs help users overcome position and time constraints to access information, it extends visual information to the third dimension, hence making spatial interactions more productive compared to 2D interfaces (Wann and Mon-Williams, 1996). With applications in varied fields like entertainment, education, and medicine, immersive experience with innovative interaction techniques can be provided through VR (Konrad et al., 2016). However, recent work in designing VR GUIs attempts to apply 2D techniques to 3D worlds, adopting interfaces from 2D GUIs (Lindeman et al., 1999). The reason for such adoption can be the challenges faced in designing VR interfaces, like distinctive visual and interaction characteristics compared to $2 \mathrm{D}$ interface design (Tanriverdi and Jacob, 2001). Real-world experiences are intuitively connected with our interaction in the $3 \mathrm{D}$ virtual world; hence, these can provide means for immersive UIs and more holistic interaction design (Steinicke et al. 2012). Interaction with VR applications and desktop 3D graphics have some explorations (Henrysson et al., 2007). These papers provide resources for usability studies in user interaction with 3D GUIs. Results mention that users can relate the spatial relationship between different objects in the real world to the augmented information provided in 3D space (Valentini, 2009). The interaction with the $3 \mathrm{D}$ models is also possible in the VR view and hence they can experience a shift from real-world to the immersive virtual world. The interactions with the VE offers many challenges and opportunities for innovation. However, when designing interfaces for such environments is considered, very few design examples exist. Developments in mobile phones offer opportunities to explore 3D and VR based gaming environments. Due to the confined physical size of mobile phones and tablets, 3D visualisation is similar to 2D and interactions are difficult (Hürst and Helder, 2011). Since VR does not have such restrictions of size, 3D GUIs need not be inspired by 2D 
GUIs, and heuristics for VR GUIs need to be designed. To design heuristics for 3D GUIs, a simple approach to design spatial number keypads in VR is followed.

Looking back into number keypad arrangements, even early calculator models used the same basic ten-key arrangement in which numbers are arranged in three rows with zero in the fourth row varying between the three columns in different arrangements (Koppa, 1985). Since calculators and mobile phones have a space constraint, this is the most effective way of arranging number keys in such a device. Whereas, in VR, an arrangement of number keypads can have different orientations in space, making use of the third dimension.

Although extensive research has been performed in the fields of VR and $3 \mathrm{D}$ interfaces, exploring opportunities in designing a spatial keypad, more specifically in the context of VR, requires the need for further study. This paper explores opportunities to study ways of designing 3D keypad interfaces. Three different interfaces are developed taking inspiration from 2D GUIs and 3D elements, and a comparison is made.

\section{Hypothesis}

Three spatial mobile keypad interfaces were developed for VR. Hypothesis tested include:

1 The circular keypad interface will have significantly more workload as compared to the other two interfaces.

2 The circular keypad interface will take significantly more time for task completion compared to the other two interfaces.

\section{Methodology}

An experiment was conducted with $n=30$ users in order to define guidelines for 3D GUIs. A user-centred design process was followed. An independent group study was performed with the three prototypes loaded in the HMD. Users were asked to perform a task of dialling a ten-digit mobile number with one of the three interfaces. Gaze pointing via head movement was used. The orientation of the interface was kept constant in space. This was done in order to replicate the real environment, as in the real world objects do not move with the user's movement. Users were given NASA-TLX workload assessment sheet after the task performance. Time taken to perform the task was calculated. Semi-structured interviews were taken to understand user's experience with the HMD and satisfaction levels.

\section{Prototypes}

Three prototypes were developed with the use of Unity3D (https://unity3d.com/unity) and Vuforia SDK (https://developer.vuforia.com/downloads/sdk) for VR. 3D models for these interfaces were developed via Google SketchUp (https://www.sketchup.com/). The design addressed important factors involved in VEs: 


\section{FOV of the HMD}

2 Orientation issues of the user

3 Utilisation of the third dimension - depth.

The first interface, the 2D keypad, includes flat 2D keys, similar to conventional mobile keypads (refer to Figure 1). Numeral data entry input was done via gaze pointer through head movement in the VR environment. Pointing at a number key by directing gaze pointer results in that number appearing on the top display panel. Feedback of number key selection is shown by a change in opacity of the same. The method was similar to pressing a number on a real mobile keypad. A green call button had to be gaze pointed upon completion of number dialling by the user.

Figure 1 2D keypad interface (see online version for colours)

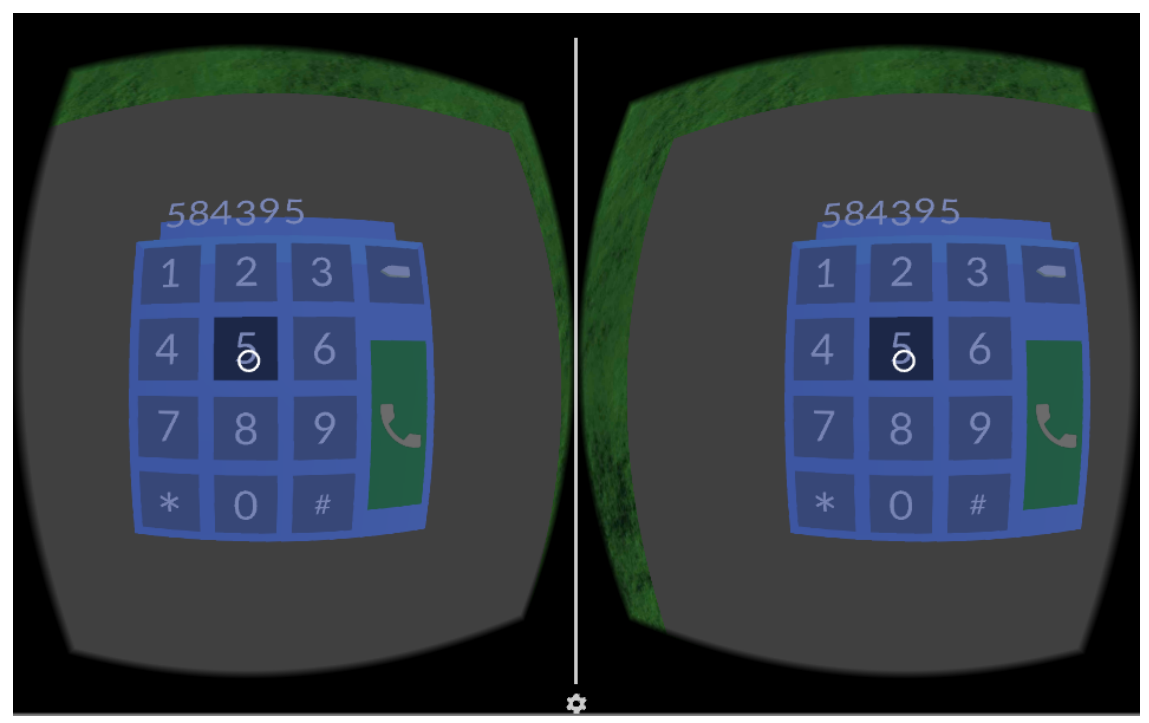

Since the first interface was very static in nature, rest two interfaces were designed to address an additional question - How do we show motion in VR GUIs?

The second interface, the cubical keypad, uses 3D cubes as keys for dialling a number (refer to Figure 2). These cubes were double line arranged in order to minimise head movement of the user and to keep all elements of the interface in a single FOV. Pointing a key (cube) using gaze pointer animates it to fall down to reach a position forming a straight line with subsequently pressed keys, all arranged in front view (dialled panel). The animation is falling down in nature and not flying up to a top panel because users relate virtual world to instances from the real world, and falling of solid objects is a natural phenomenon. Once this animation is complete, the number key (cube) reappears in its position to incorporate repeated entry. A call button is present in line with the above arrangement, pointing at which indicates completion of number dialling task. 
Figure 2 Cubes keypad interface (see online version for colours)

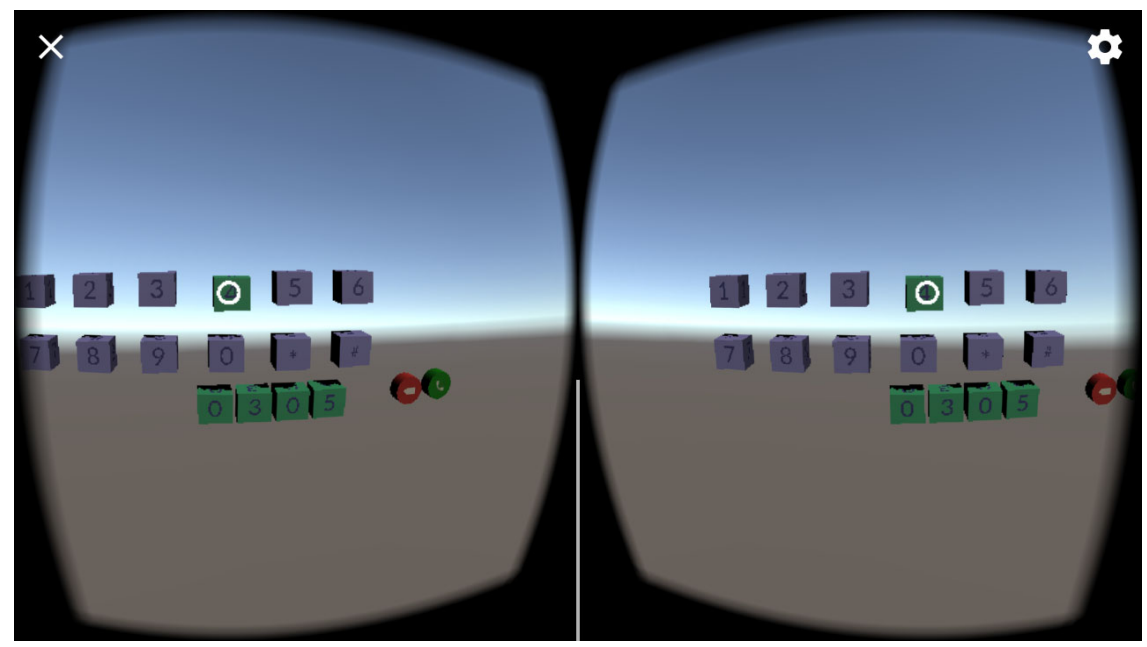

The third interface, the circular keypad, uses spheres as keys for dialling a number (refer to Figure 3). The arrangement of these spheres is done in a circular manner with the call button and back button placed in the midline. A circular arrangement is done in order to reduce the error of gaze pointing to an undesirable number key in the process of passing over it to reach the desired key, which is very much possible in matrix-like an arrangement. In this interface, pointing a key (sphere) animates it to reach a top panel similar to a mobile keypad where the dialled number appears on the top. Once this animation is complete, the number key (sphere) reappears in its position to incorporate repeated entry.

Figure 3 Circular keypad interface (see online version for colours)

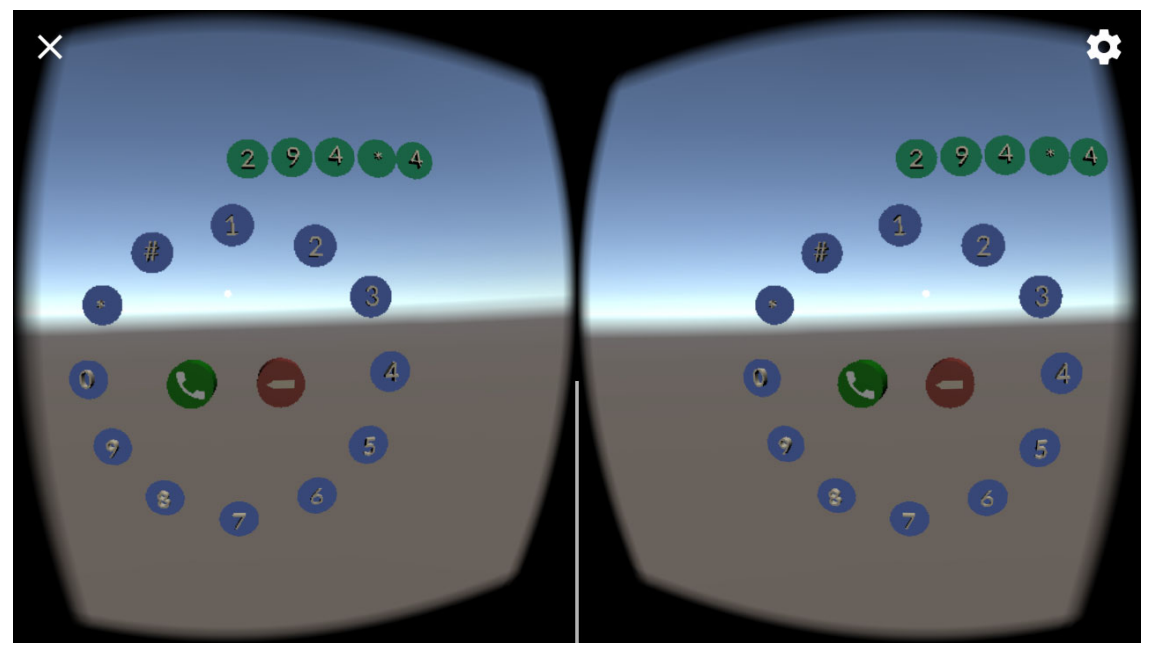

These interfaces were tested with students. User studies performed are mentioned in the next section. All three interfaces are differently designed to test different interactions and draw conclusions. 


\section{User study}

An independent user group study was conducted amongst 30 participant users of age group 18-22 years, all undergraduate students. The users were familiar with mobile phone dialling system and were proficient in interacting with smartphones. All these users were new to the VR environment, hence at the beginner proficiency level of VR interactions.

\subsection{Experiment design}

Independent variables include three VR interfaces of spatial mobile keypad. Dependent variables include:

1 time

2 workload

3 perceived ease of use and usefulness.

Instruments include:

1 clock

2 questionnaire based on SUS and TAM model

3 VR headset.

\subsection{Experiment process}

The participants were briefed about the functioning of the prototypes at the beginning of the experiment. Scenarios describing use-cases of the application of our prototypes and gaze pointer method of input was explained. The experiment was conducted in three groups of ten participants each. Each group was tested with one VR keypad interface. Each participant was initially asked to dial a phone number in a conventional mobile keypad (this was done so that all users have the same reference), following a task of dialling a ten-digit mobile phone number, in the VR keypad interface allotted to him $/ \mathrm{her}$, in a single trial. The same phone number was given to all participants during their respective task. After completion of the task, NASA-TLX workload assessment sheet was filled through pen and paper. Post this, a questionnaire of perceived ease of use and perceived usefulness was rated on a Likert scale of $1-5(1=$ strongly disagree, $5=$ strongly agree). Semi-structured interviews were also taken from the users.

\section{Findings}

Perceived ease of use and usefulness: Each participant filled a questionnaire based on SUS and TAM model on a Likert scale of 1-5 $(1=$ strongly disagree, $5=$ strongly agree $)$. Semi-structured interviews were conducted to get user feedback on the interface and understand user satisfaction level. Figure 4 represents the mean and standard deviation of the perceived usefulness of the three VR keypad interfaces. Perceived usefulness tested the following parameters: 
1 Did the user have control of the task?

2 Can user accomplish the task quickly using the interface?

3 Does it increase productivity?

4 Does the interface make user's task easier?

5 Was the interface useful in performing the task

The mean of perceived usefulness of 2D keypad is maximum since users find it useful to use an interface similar to conventional mobile keypads. There is less learning involved in this case. Some users also stated that there are chances of error in the 2D and cubes keypad interface when shifting from one number key to the other since the gaze passes through other number keys in the path.

Figure 4 Perceived usefulness (see online version for colours)

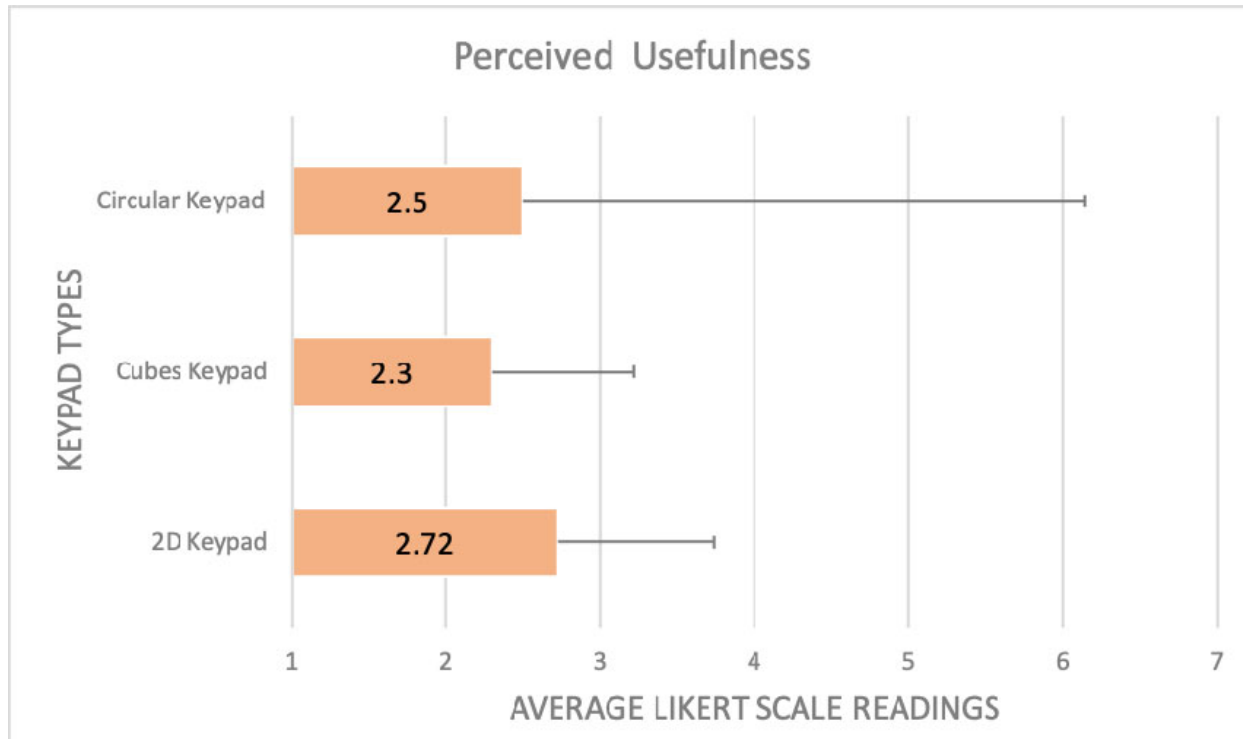

Figure 5 represents the mean and standard deviation of the perceived ease of use of the three VR keypad interfaces. Perceived ease of use tested the following parameters:

1 How frequently would the user use the interface?

2 Is a technical person required?

3 Will people quickly learn the interface?

4 Is the interface cumbersome to use?

The mean of perceived ease of use of the circular keypad interface is more than the other two keypad interfaces. Some users stated that circular arrangement reduced error as they had enough space to move from one number key to the other. Although, some felt circular arrangement increased their head movement during the task. 
Figure 5 Perceived ease of use (see online version for colours)

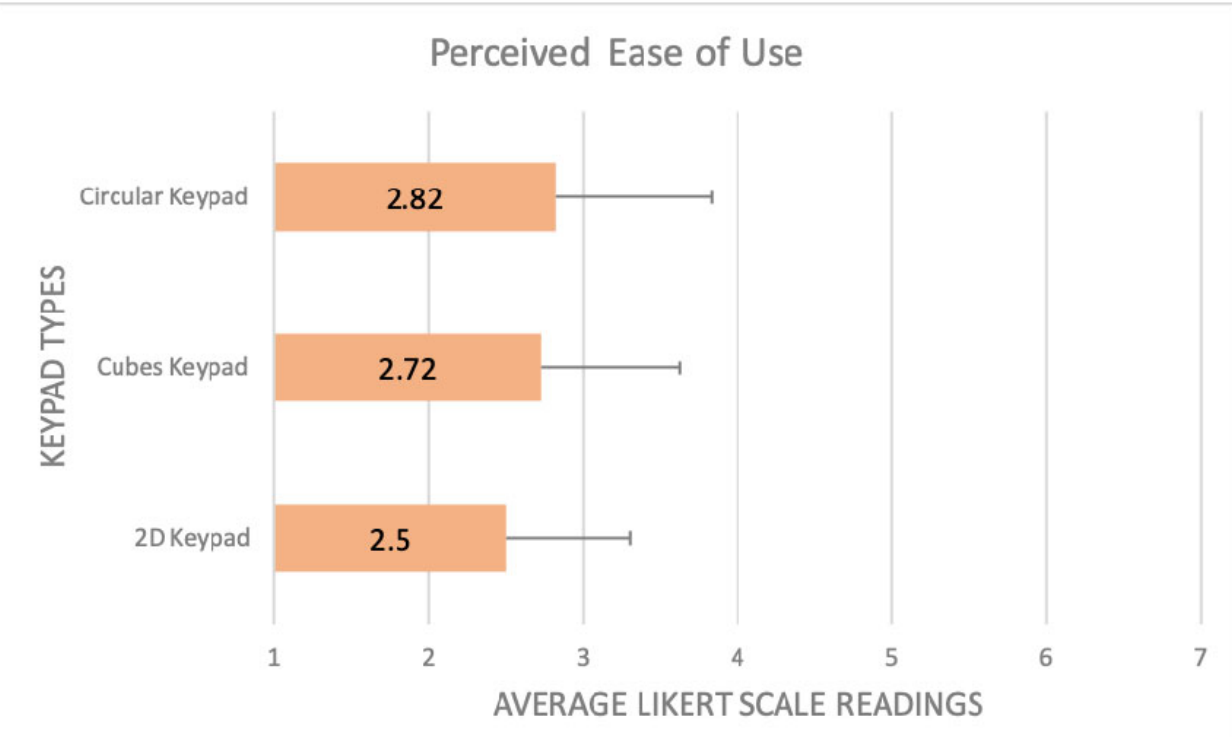

Workload: NASA-TLX workload assessment was done immediately after task performance. The calculation was done as per followed by NASA-TLX, and mean WWL score of each participant was calculated (refer to Figure 6). The data was found normally distributed. Following t-tests show that workload comparison between circular and cubes keypad interface has a $p=0.105$ and $p=0.109$ respectively. Since this is a two-tailed value, single tailed result and a large effect size $d=0.8055$ show that workload of circular keypad interface is significantly more than that of cubes keypad interface. The comparison of the workload of circular keypad interface with the 2D keypad interface shows an effect size $d=0.705$. Although there is no significant difference in the overall mean WWL score of both the interfaces considering $p>0.05$, since the effect size is moderate, we can conclude that workload of circular keypad interface is significantly more than that of 2D keypad interface. Effect size is important to note since it allows to make a decision when the number of participants are less in an independent group study. Hence, circular keypad interface has significantly more workload compared to the other two interfaces. This can be because of increased head movement due to the circular arrangement of number keys.

Task completion time: The time taken to complete the task of dialling a ten-digit phone number in each VR interface was calculated from screen recordings. The data was found normally distributed. Independent sample t-tests showed that the mean time taken for task performance in circular keypad interface is significantly more than the time taken in the other two VR interfaces $(p<0.05)$. Although a significant difference in mean time taken for task performance in Cubes keypad interface and 2D keypad interface is not found, the effect size is found to be $d=0.653$. The effect size matters when the study is conducted with a small group of participants. Hence, due to the smaller number of participants and 
moderate effect size, we can conclude that the mean time taken in cubes keypad interface is more than that in 2D keypad interface. This might be due to an initial learning curve involved in the cubes keypad interface, as the users are not used to such an arrangement of number keys.

Figure 6 NASA TLX workload analysis (see online version for colours)

NASA TLX readings

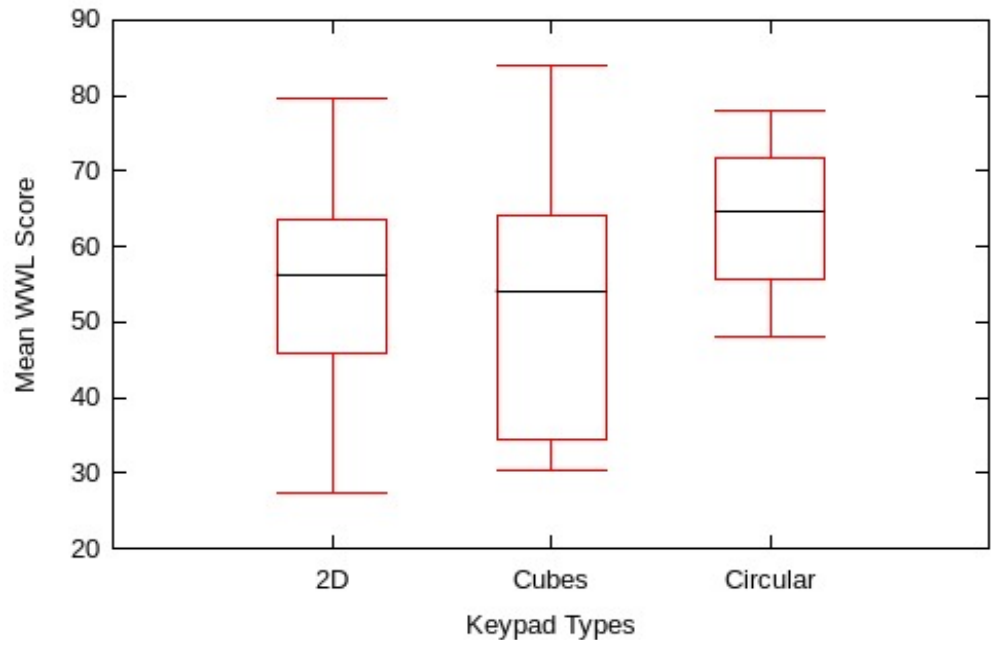

Figure 7 Task completion time per participant (see online version for colours)

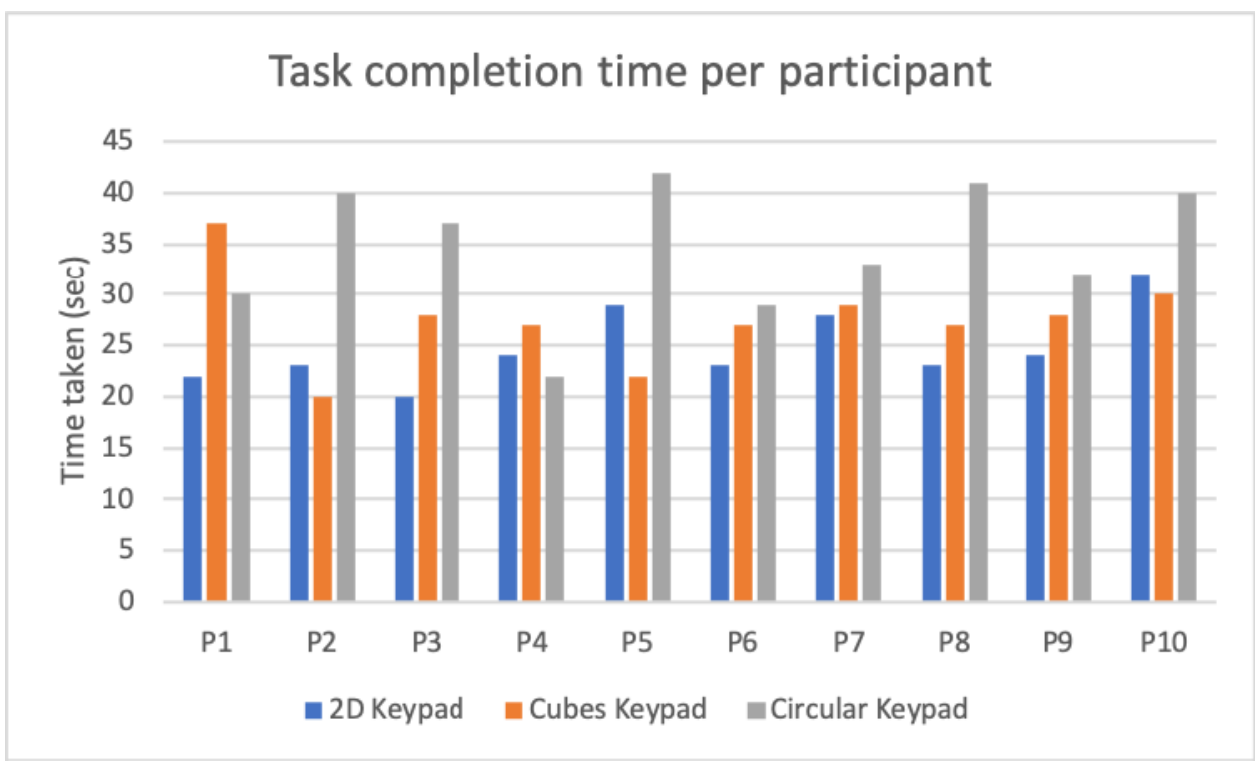


Figure 8 Average task completion time (see online version for colours)

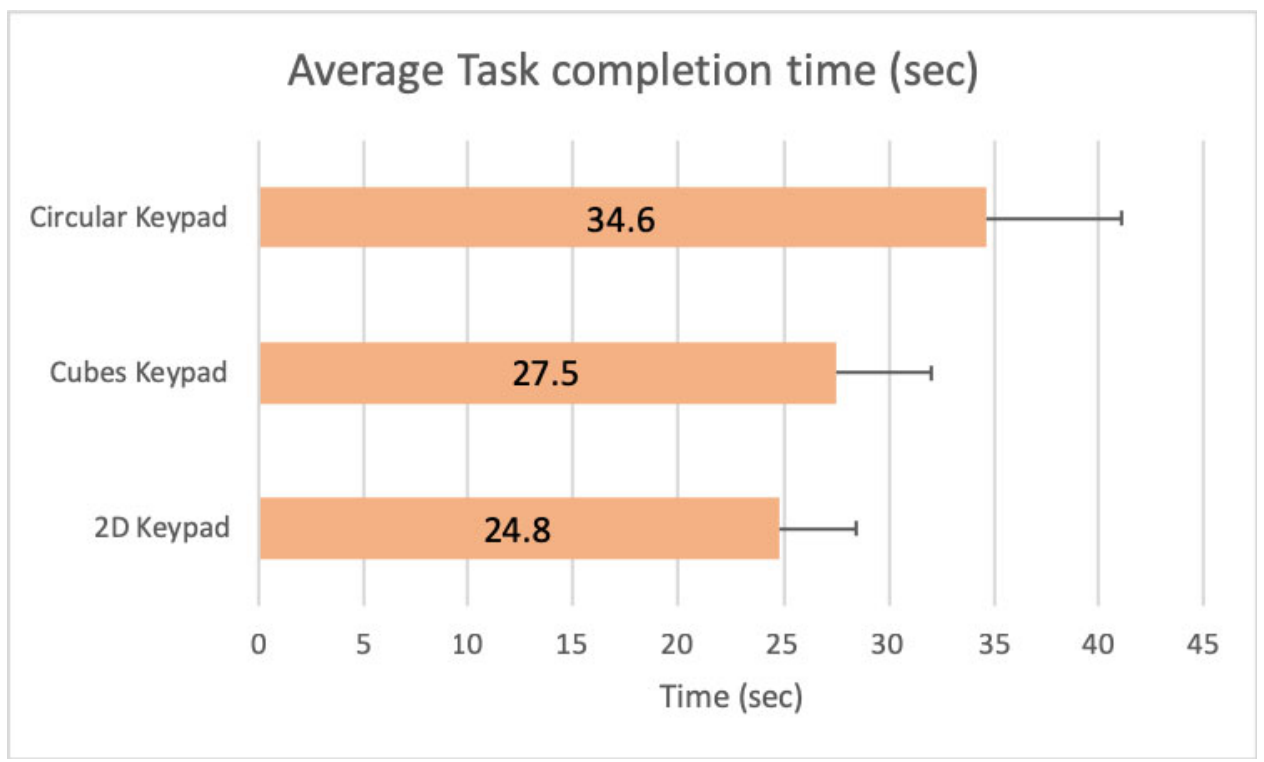

\section{Discussions}

Different ergonomic factors are involved in the design of 3D GUIs, including problems associated with HMDs and VEs. In order to build guidelines for reducing cognitive load of such interfaces, several factors need to be considered. Questions about the aspect ratio of the virtual interface also arise. In a $2 \mathrm{D}$ interface, the aspect ratio is confined to the screen, but in a 3D space, what would be those dimensions? Should the real environment be taken as a reference for deciding the dimensions of a virtual space? Design of 3D GUIs pose questions about different human factors involved in the interaction and further study is going on to enhance the user experience in a VE.

This study explains the role of four factors majorly: task-completion time, perceived ease of use, perceived usefulness and cognitive load. It is concluded that the circular keypad interface has the maximum workload compared to the other two, since it involves lot of head movement with the current head-gaze input method. Users took significantly more time to enter a number in the circular interface as well compared to the other two. Hence, we can conclude that although the circular interface was designed to reduce errors, it had a huge negative impact on the users. 3D GUI designers need to consider that although VR offers a lot of space for 3D design and animations, not all the space is always visible to the user and appropriate input methods need to be used to design such scenarios. The task completion time is also minimum in case of $2 \mathrm{D}$ keypad as it is a known interface to our users. In order to switch new users to adapt to VEs, a smooth transition needs to be done. 
The designer of 3D GUIs may not be able to rely on heuristics of interactions as prevalent in 2D interfaces of the real-world, hence new heuristics will have to be developed for future 3D interactive panels in VR environment. Further, since the 3D virtual space increases the real estate for interaction, issues like the variable of 'motion and direction' in addition with speed of response time arise. These issues need to be considered in designing heuristics for 3D GUIs. In scenarios where user's hands are engaged while interacting in VEs, gaze pointing can be highly helpful. Users should be allowed to control the duration of the functionality of the gaze pointer as per convenience. This would provide ease of use in interaction and reduce the time to switch or select elements in 3D space. 3D VR interface if appropriately designed may find acceptance and rapid adaptation amongst users who are so far used to $2 \mathrm{D}$ interfaces. User perception towards 3D interface is very positive and enthusiastic. Users' inclination towards gesture-based interactions and eye tracking inputs is seen. Further study is going on to incorporate gaze as well as gesture-based interactions based on which design heuristics for 3D GUIs will be developed.

\section{References}

'3D Design Software: 3D Modeling on the Web', SketchUp [online] www.sketchup.com/.

Henrysson, A., Marshall, J. and Billinghurst, M. (2007) 'Experiments in 3D interaction for mobile phone AR', in Proceedings of the 5th International Conference on Computer Graphics and Interactive Techniques in AUSTRALIA and Southeast Asia, December, ACM, pp.187-194.

Hürst, W. and Helder, M. (2011, November) 'Mobile 3D graphics and virtual reality interaction', in Proceedings of the 8th International Conference on Advances in Computer Entertainment Technology, November, ACM, p.28.

Konrad, R., Cooper, E.A. and Wetzstein, G. (2016) 'Novel optical configurations for virtual reality: evaluating user preference and performance with focus-tunable and monovision near-eye displays', in Proceedings of the 2016 CHI Conference on Human Factors in Computing Systems, May, ACM, pp.1211-1220.

Koppa, R.J. (1985) 'A study of data entry keyboards: the 4 x 4 keypad', Texas Transportation Inst. College Station.

Lindeman, R.W., Sibert, J.L. and Hahn, J.K. (1999) 'Towards usable VR: an empirical study of user interfaces for immersive virtual environments', in Proceedings of the SIGCHI conference on Human Factors in Computing Systems, May, ACM, pp.64-71.

Nichols, S. (1999) 'Physical ergonomics of virtual environment use', Applied Ergonomics, Vol. 30, No. 1 , pp.79-90.

Steinicke, F., Benko, H., Krüger, A., Keefe, D., de la Riviére, J.B., Anderson, K. and Pakanen, M. (2012) 'The 3rd dimension of CHI (3DCHI): touching and designing 3D user interfaces', in CHI'12 Extended Abstracts on Human Factors in Computing Systems, May, ACM, pp.2695-2698.

Tanriverdi, V. and Jacob, R.J. (2001) 'VRID: a design model and methodology for developing virtual reality interfaces', in Proceedings of the ACM symposium on Virtual Reality Software and Technology, November, ACM, pp.175-182.

Unity Technologies 'Products' [online] unity3d.com/unity.

Valentini, P.P. (2009) 'Interactive virtual assembling in augmented reality', International Journal on Interactive Design and Manufacturing (IJIDeM), Vol. 3, No. 2, pp.109-119.

Vuforia Developer Portal 'Vuforia Engine 8.3, SDK Download [online] developer.vuforia.com/downloads/sdk. 
Wann, J. and Mon-Williams, M. (1996) 'What does virtual reality NEED?: human factors issues in the design of three-dimensional computer environments', International Journal of HumanComputer Studies, Vol. 44, No. 6, pp.829-847.

Wilson, J.R. (1997) 'Virtual environments and ergonomics: needs and opportunities', Ergonomics, Vol. 40, No. 10, pp.1057-1077.

Wilson, J.R. (1999) 'Virtual environments applications and applied ergonomics', Applied Ergonomics, Vol. 30, No. 1, pp.3-9. 